\title{
Soil environmental factors shape the rhizosphere arbuscular mycorrhizal fungal communities in South African indigenous legumes (Fabaceae)
}

\author{
AFOLAKEMI ABIBAT ALIMI ${ }^{1, \bullet}$, RASHEED ADELEKE ${ }^{2}$, ANNAH MOTEETEE ${ }^{3}$ \\ ${ }^{1}$ Department of Botany and Plant Biotechnology, University of Johannesburg. PO Box 524, Auckland Park, 2006, Johannesburg, Gauteng, South Africa \\ Tel.: +27-64-0868188, •email: habbyfol@yahoo.com \\ ${ }^{2}$ Unit for Environmental Sciences and Management, North West University. Potchefstroom campus, Potchefstroom 2520, North West Province, South \\ Africa \\ ${ }^{3}$ Department of Botany and Plant Biotechnology, University of Johannesburg. PO Box 524, Auckland Park, 2006, Johannesburg, Gauteng, South Africa
}

Manuscript received: 8 March 2021. Revision accepted: 6 April 2021.

\begin{abstract}
Alimi AA, Adeleke R, Moteetee A. 2021. Soil environmental factors shape the rhizosphere arbuscular mycorrhizal fungal communities in South African indigenous legumes (Fabaceae). Biodiversitas 22: 2466-2476. The crucial role played by arbuscular mycorrhizal fungi (AMF) in the nutritional adaptation of plants to nutrient-deficient soils is well recognized. However, little is known about the diversity of AM fungal communities in the rhizosphere of indigenous legumes of South Africa. This study presents the first morphological characterization of the AM fungal diversity present in the rhizosphere of eleven indigenous legume species from two provinces in South Africa and correlates the diversity and community structure of AMF to soil physicochemical properties. Twenty AM fungal species belonging to ten genera were identified; Glomus and Acaulospora were the dominant genera, while Acaulospora colombiana, A. mellea, and Claroideoglomus etunicatum were the prevalent species. The AM fungal spore density, diversity, and community structure differed markedly among the legume species in both provinces. Correlation and canonical correspondence analyses revealed that the diversity and spatial structure of AM fungal communities were significantly influenced by soil properties. This study provides a theoretical insight into the future potential of using the dominant AM fungal species as inoculants for sustainable management of legumes and indicated that soil factors are important environmental determinants of AM fungal diversity and community structure.
\end{abstract}

Keywords: Arbuscular mycorrhizal fungi, community structure, diversity, indigenous legumes, soil factors

Abbreviations: AMF: Arbuscular mycorrhizal fungi, P: available phosphorus, K: available potassium, $\mathrm{OC}^{-}$organic carbon, $\mathrm{NO}_{3}^{-}-\mathrm{N}$ : nitrate, $\mathrm{NH}_{4}{ }^{+}-\mathrm{N}$ : ammonium, $\mathrm{Cu}$ : copper $\mathrm{Mn}$ : manganese, $\mathrm{Zn}$ : zinc BD: bulk density, SD: spore density, SR: species richness, IF: isolation frequency, RA: relative abundance, H: Shannon-Weiner diversity index, J: Pielou evenness index, CCA: canonical correspondence analysis

\section{INTRODUCTION}

Legumes (Fabaceae) constitute the second most important plant family after grasses (family Poaceae), contributing significantly to global food and nutritional security (Graham and Vance 2003). Additionally, legumes are considered essential service providers for natural and agroecosystems due to their ability to contribute to soil fertility through biological nitrogen fixation (Peoples and Craswell 1992; Cleveland et al. 1999). Legumes establish mutualistic relationships with arbuscular mycorrhizal fungi (AMF), which help them to meet their nutritional requirements for phosphorus $(\mathrm{P})$ from soils with low $\mathrm{P}$ availability (Chalk et al. 2006) and promote their tolerance to several biotic and abiotic stresses (Smith et al. 2010). Such AM fungal-mediated supply of $\mathrm{P}$ is essential in driving the high P-demanding legume-rhizobia symbiosis, thus making legumes highly reliant on the arbuscular mycorrhizal association than other plant groups (Barea et al. 1987).
The AMF are ubiquitous in terrestrial ecosystems, where they form obligate symbiotic relationships with the roots of vast majority of land plants, including legumes (Brundrett and Tedersoo 2018). Nevertheless, it has been established that the diversity and structure of rhizosphere AM fungal communities vary in different plants, and that host plant species and soil characteristics are strong determinants of this differentiation (Eom et al. 2000; Johnson et al. 2004; Martínez-García et al. 2011; SantosGonzález et al. 2011; Liu et al. 2015).

South Africa is marked by acidic soils with very low nutrient concentrations, particularly phosphorus (Barnard and du Preez 2004; Hawkins et al. 2005). That notwithstanding, the country is characterized by a high diversity of indigenous legumes, which are widely distributed in different biomes (Trytsman et al. 2011). Under these soil nutrient-deficient conditions, it is envisaged that AMF are likely to play a vital role in the nutrition and adaptation of indigenous legumes to this environment. However, the diversity of indigenous AM fungal communities present in the rhizosphere of these 
plants remains unexplored. Indeed, there is an urgent need for the establishment of conservation strategies as almost $30 \%$ of indigenous South African legumes are currently threatened due to several anthropogenic factors (Yahara et al. 2013).

One promising approach that is currently emphasized for the propagation and management of indigenous plant communities is the use of indigenous AMF from the soil (Hawley and Dames 2004; Fitzsimons and Miller 2010). Indigenous AMF enhances host plants' uptake of mineral nutrients and water from soils, improve soil aggregate stability through the release of glomalin into the soil, and are also well adapted to the biotic and abiotic stress conditions that characterize the local environments of their indigenous hosts (de Oliveira et al. 2017). The combination of these attributes can facilitate plant growth, performance, and survival in stressed environments, and hence may benefit sustainable management programs (Maltz and Treseder 2015). Consequently, the knowledge of the indigenous AM fungal diversity in the rhizosphere is a critical fundamental step towards the understanding of the functional roles of AMF in natural ecosystems. Such information would enable the identification and selection of efficient AM fungal species or species combinations that can be exploited as inoculants for the management of plants under both nursery and field conditions (Chen et al. 2018). Therefore, the aim of this study was firstly to assess the diversity of AM fungal communities present in the rhizosphere of eleven indigenous legumes from two South African provinces, using morphological method, and secondly to investigate the influence of soil properties on the diversity and spatial structure of AM fungal communities.

\section{MATERIALS AND METHODS}

\section{Study area}

The study was conducted in certain areas within the Gauteng and Mpumalanga Provinces (Figure 1). These provinces are located in the grassland biome of South Africa and have summer rainfall (Rutherford and Westfall 1986). Grasses dominate the vegetation of the study areas, but a variety of forbs and few trees are also present (Mucina et al. 2006; Lötter, 2014). There is a broad spectrum of soil types, including the red-yellow-grey latosol plinthic catena, black and red clays and solonetzic soils, freely drained latosols, and black clays (Rutherford and Westfall 1986). The Gauteng Province is situated on latitude $26.2708^{\circ} \mathrm{S}$ and longitude $28.1123^{\circ} \mathrm{E}$, at an average altitude of $1,512 \mathrm{~m}$ above sea level. The average minimum and maximum temperature range from $10.2{ }^{\circ} \mathrm{C}$ to $24.8{ }^{\circ} \mathrm{C}$, and annual precipitation averages $771 \mathrm{~mm}$ per year. Mpumalanga is positioned on latitude $-29.8129^{\circ} \mathrm{S}$ and longitude $30.6364^{\circ} \mathrm{E}$. The province has several distinctive physiographic regions; the Highveld, where the altitude ranges from 1,200-1,800 $\mathrm{m}$ above sea level in the west; the forested Drakensberg mountains rising to more than 2,300 $m$ above sea level in the east; and the low-lying Lowveld in the northeast. Temperatures in Mpumalanga vary with altitude, from a mean of $10{ }^{\circ} \mathrm{C}$ in the Highveld and an average of $23{ }^{\circ} \mathrm{C}$ in the subtropical Lowveld. The annual rainfall increases from west to east, averaging 341-1933 $\mathrm{mm}$.

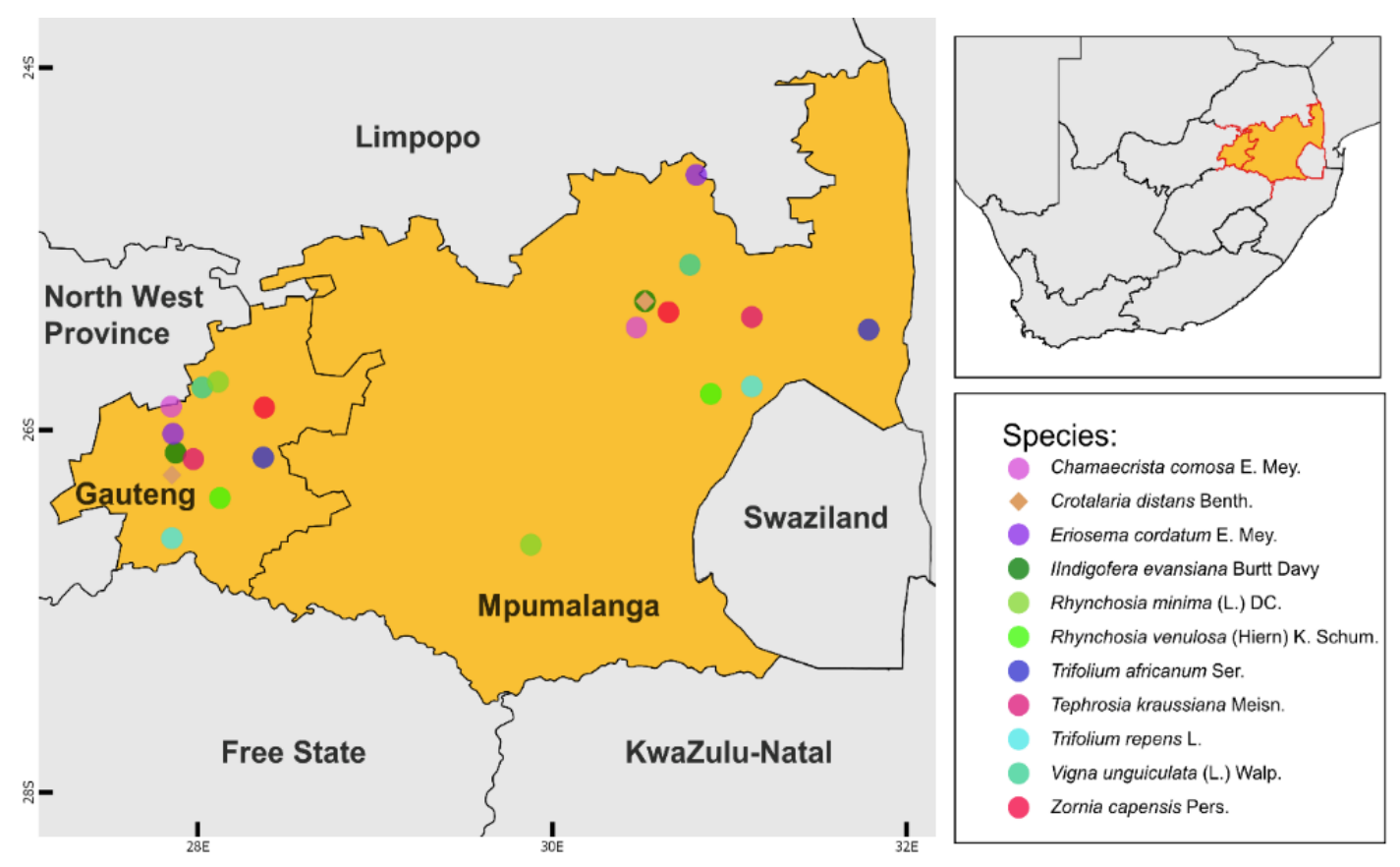

Figure 1. Map of South Africa showing the sampling points of legumes in each province 


\section{Procedures \\ Sampling}

Sampling was conducted in February 2019. In each province, rhizosphere soils were aseptically collected from four individuals of each legume species at a depth of 0-20 $\mathrm{cm}$ using a soil auger (Eijkelkamp Soil and Water, EM Giesbeek, Netherlands). Additionally, cylindrical soil cores were collected per sample with a bulk density sampler (Eijkelkamp Soil and Water, EM Giesbeek, Netherlands), to calculate soil bulk density. Samples were kept in sterile Ziplock bags and transported to the laboratory. A subsample was stored at $4{ }^{\circ} \mathrm{C}$ prior to spore isolation, while the other fraction was air-dried, passed through a $2 \mathrm{~mm}$ sieve, and used for the determination of soil physicochemical properties.

\section{Soil analysis}

Selected physicochemical properties of soil were analyzed following standard protocols. Briefly, soil $\mathrm{pH}$ was determined from a 1:2.5 (w/v) soil suspension in $1 \mathrm{~N} \mathrm{KCl}$. Available potassium $(\mathrm{K})$ was assessed using the ammonium acetate method of Schollenberger and Simon (1945). Organic carbon (OC) was quantified using the WalkleyBlack method (Walkley and Black 1934). Available phosphorus (P) was evaluated using the Bray 1 method (Bray and Kurtz 1945). Nitrate $\left(\mathrm{NO}_{3}^{-}-\mathrm{N}\right)$ was determined by the method of Sonneveld and Van den Ende (1971), while ammonium $\left(\mathrm{NH}_{4}{ }^{+}-\mathrm{N}\right)$ was estimated using the ammoniaselective electrode method (Banwart et al. 1972). Extractable micronutrients including copper $(\mathrm{Cu})$, manganese $(\mathrm{Mn})$, and Zinc $(\mathrm{Zn})$ were obtained by acid digestion of soil (Jackson 1958). Bulk density (BD) was measured after drying soil samples for $48 \mathrm{hrs}$ at $105{ }^{\circ} \mathrm{C}$ (ISO 2017) and soil particle size distribution was determined with the Bouyoucos hydrometer method (Bouyoucos 1962).

\section{AM fungal spore isolation and identification}

Spores were isolated from replicates of each legume species using the modified wet sieving and decanting method (Brundrett et al. 1996) and sucrose density gradient centrifugation method (Daniels and Skipper 1982). Summarily, $1000 \mathrm{ml}$ of water was added to $100 \mathrm{~g}$ of airdried soil. The mixture was stirred, and the resulting soil suspension was decanted through a series of 750, 250, 100, and $38 \mu \mathrm{m}$ sieves arranged in decreasing order of mesh sizes. The materials retained in the $750 \mu \mathrm{m}$ sieve was checked for large spores and sporocarps. Soil materials retained in the 250,100 , and $38 \mu \mathrm{m}$ sieves were washed into centrifuge tubes. The tubes were centrifuged at 4,000 rpm for 5 mins. Thereafter, the supernatants were decanted, $50 \%(\mathrm{w} / \mathrm{v})$ sucrose solution was added to the pellets, mixed gently, and centrifuged at 3,000 rpm for $1 \mathrm{~min}$. The supernatants from each tube were then decanted into the 38 $\mu \mathrm{m}$ sieve and rinsed with distilled water several times to remove the sucrose solution. Recovered spores and sporocarps were washed into sterile Petri dishes and counted under the dissecting microscope at 50x magnification. Spore density (SD) was expressed as the total number of spores and sporocarps in $100 \mathrm{~g}$ of soil. For identification, spores were picked under the dissecting microscope with glass micropipette, mounted on slides with polyvinyl-lactic acid-glycerol (PVLG) or polyvinyllactic acid-glycerol mixed with Melzer's reagent (1:1 (v/v) and observed at 100-400x magnification. The spores were identified to the species level on the basis of shape, color, size, number of spore walls, and presence or absence of subtending hyphae using the taxonomic criteria described by Schenck and Perez (1990), together with the descriptions of reference cultures from the International Culture Collection of (vesicular) arbuscular mycorrhizal fungi INVAM (1997).

\section{Arbuscular mycorrhizal fungal diversity and community studies}

Species richness (SR), isolation frequency (IF), relative abundance (RA) were used to evaluate the structure of AM fungal communities in the rhizosphere of different legumes. These indices were computed as follows: Species richness (SR) was measured as the total number of identified AMF species per soil sample. Isolation frequency $(\mathrm{IF})=$ (the number of samples in which a particular AMF species was observed/the total number of samples) $\times 100$, where AMF species were classified into the following groups according to Zhang et al. (2004): dominant (IF > $50 \%$ ), most common (IF 31\%-50\%), common (IF 10\%$30 \%)$, and rare $(\mathrm{IF}<10 \%)$. Relative abundance $(\mathrm{RA})=($ the number of spores of a particular genus)/total number of identified spores $) \times 100$. The AM fungal species diversity in different legumes was assessed by the Shannon-Weiner diversity index, H (Shannon 1948) using the formula, $\mathrm{H}=-$ $\Sigma_{\mathrm{i}}^{\mathrm{k}}=1(P i \ln P i) ; P i$ is the relative abundance of each identified AMF species per sample and is calculated by the formula $\mathrm{Pi}=n i / N$, where $n i$ represents the number of individuals of a species and $N$ is the total number of individuals of all species. Species evenness was obtained by Pielou evenness index, J (Pielou 1966) using the formula, $J=H / \log (S)$, where $H$ is the value obtained for Shannon-Weiner index and $\mathrm{S}$ is the species richness.

\section{Statistical analysis}

Statistical analyses were performed using $\mathrm{R}$ statistical software version 4.0.0. (R Core Team, 2020). Significant differences in SD, SR, H, J, and soil properties among plants within and between provinces were tested using Two-way ANOVA followed by Tukey HSD (Tukey Honest Significant Differences). The data were tested for normality and transformation was attempted if necessary, prior to analysis of variance. Significant means were compared using Least Significant Difference (LSD) at the $5 \%$ probability level $(P<0.05)$. Pearson correlation analysis was used to test for the relationship between AM fungal spore density, diversity indices, and soil properties. The canonical correspondence analysis (CCA) was employed to evaluate the influence of soil parameters on the spatial structuring of AMF communities. For the CCA analysis, soil and AMF community data were logtransformed using an automatic forward and backward Stepwise model in the vegan package. Test for significance of the environmental (constraining) variables were checked using a permutation test. 


\section{RESULTS AND DISCUSSION}

\section{Soil properties}

Results of the rhizosphere soil physicochemical parameters of studied plants from the two provinces are shown in Table 1 . Generally, the soils were acidic $(\mathrm{pH}<7)$ and low in nutrients. The mean soil $\mathrm{pH}$ ranged from 4.385.87 in Gauteng and 4.48-5.84 in Mpumalanga. The textural classification of rhizosphere soils of legumes from Gauteng was largely sandy-loam, while in Mpumalanga, the rhizosphere soils were characterised by a broad spectrum of textural types including sandy-loam, sandy clay, sandy clay loam, loam, clay, loamy sand, and clay loam. Except for bulk density, all the measured soil parameters varied significantly $(P<0.05)$ among plants both within and between provinces.

\section{Arbuscular mycorrhizal fungal spore density, diversity, and community structure}

The mean AM fungal spore density isolated from the rhizosphere of studied plants ranged from 306-812 in Gauteng and 284-759 in Mpumalanga (Table 2). In both provinces, the highest and lowest average SD was observed in Trifolium repens and Tephrosia kraussiana, respectively. Spore density differed significantly $(P<0.05)$ among legume species within provinces, but no significant difference $(P>0.05)$ was observed in SD of the same legume species between provinces. Of all the diversity parameters (SR, H, and J) investigated, only SR and $\mathrm{H}$ differed significantly $(P<0.05)$ among legumes within provinces, but no significant difference $(P>0.05)$ was found between provinces (Table 2).

Twenty species of AMF were identified in the rhizosphere of legumes in both provinces (Figure 2). These species are distributed within ten genera. Glomus was the predominant genus (represented by seven species), Acaulospora (three species), Rhizophagus and Septoglomus (two species each), and Claroideoglomus, Entrophospora, Funneliformis, Sieverdingia, Gigaspora, and Scutellospora (one species each). Based on IF, Acaulospora colombiana, Claroideoglomus etunicatum, A. mellea, Sieverdingia tortuosa, Glomus ambisporum, and Rhizophagus intraradices were the dominant species in the rhizosphere of legumes in Gauteng, whereas A. mellea, C. etunicatum, $G$. ambisporum, G. magnicaule, A. colombiana, and Septoglomus constrictum were the dominant species in Mpumalanga. Acaulospora colombiana, A. mellea, and $C$. etunicatum were the most frequent species since they were present in all samples, with the highest IF of $100 \%$. Gigaspora decipiens and Scuttelospora biornata were the rare AM fungal species in the rhizosphere of legumes in both provinces.

The AM fungal community structure and their relative abundance varied among legumes within and between provinces. In Gauteng, the genus Glomus was the most abundant in the rhizosphere of $C$. distans, I. evansiana, $T$. africanum, T. repens, and Z. capensis; Acaulospora exhibited higher abundance in $C$. comosa, $R$. minima, and V. unguiculata; Septoglomus was the most abundant genus in E. cordatum and $R$. venulosa, while the genus Funneliformis had higher abundance in T. kraussiana. In Mpumalanga, Glomus was the most abundant genus in $C$. comosa, R. minima, and T. africanum, whereas genus Acaulospora was the most abundant in $C$. distans, E. cordatum, I. evansiana, $R$. venulosa, T. krausiana, T. repens, V. unguiculata, and Z. capensis.

\section{Influence of soil factors on AM fungal diversity and community structure}

Pearson correlation analysis showed that SD, SR, H, and $\mathrm{J}$ were positively correlated with soil $\mathrm{pH}$; $\mathrm{SD}$ was negatively correlated with $\mathrm{NO}_{3}$ but positively correlated with Mn; SD and SR were negatively correlated with $\mathrm{P}$ but positively correlated with $\mathrm{K}$. Also, a significant positive correlation was found between SR, H, J, and BD (Table 3). The step-wise model for the CCA triplot revealed that soil properties significantly $(P=0.001)$ influenced the structure of AM fungal communities (Figure 3 ). The first and second CCA axes showed that more than $50 \%$ of the total variability in AMF community structure was explained by soil variables. According to the CCA results, the distribution of $G$. delhiense, G. tubaeforme, G. sinuosum, $G$. taiwanensis, $G$. decipiens, and $S$. biornata were significantly associated with $\mathrm{K}$ and $\mathrm{Zn}$, the distribution of $S$. deserticola was correlated with $\mathrm{BD}$, the distribution of $A$. colombiana and G. magnicule were related with $\mathrm{P}_{\text {and }} \mathrm{NH}_{4}$, whereas the distribution of $R$. intraradices, $G$. rubiforme, $R$. irregularis, $E$. infrequens, $F$. geosporum, and $S$. constrictum were least influenced by soil properties.

\section{Discussion}

Most members of the family Fabaceae form AM symbiosis, although mycorrhizal dependency varies among species (Trappe 1987). Similar to findings from previous studies (Wang et al. 2004; Wang and Jiang 2015; Toh et al. 2018), there was a significant difference in spore density among legume species in this study. This may be due to the composition and proportion of root exudates secreted by different host plants (Carrenho et al. 2002; Jones et al. 2004). In natural ecosystem, different legumes produce different types of flavonoids and strigolactones, which act as chemo-attractants for AMF to the roots, and ultimately influence AMF growth and spore production (D'arcyLameta 1988; Steinkellner et al. 2007). Other factors like differences in sporulation patterns among AM fungal taxa and environmental influences can equally contribute to variation in AMF spore populations (Anderson et al. 1984; Addy et al. 1994; Eom et al. 2000). 
Table 1. Physico-chemical properties of soil in the rhizosphere of legumes in both provinces

\begin{tabular}{|c|c|c|c|c|c|c|c|c|c|c|c|c|}
\hline \multirow[t]{15}{*}{ Gauteng } & Soil properties & $\begin{array}{l}\text { Chamaecrista } \\
\text { comosa }\end{array}$ & $\begin{array}{l}\text { Crotalaria } \\
\text { distans }\end{array}$ & $\begin{array}{l}\text { Eriosema } \\
\text { cordatum }\end{array}$ & $\begin{array}{l}\text { Indigofera } \\
\text { evansiana }\end{array}$ & $\begin{array}{l}\text { Rhynchosia } \\
\text { minima }\end{array}$ & $\begin{array}{l}\text { Rhynchosia } \\
\text { venulosa }\end{array}$ & $\begin{array}{l}\text { Trifolium } \\
\text { africanum }\end{array}$ & $\begin{array}{l}\text { Tephrosia } \\
\text { kraussiana }\end{array}$ & $\begin{array}{l}\text { Trifolium } \\
\text { repens }\end{array}$ & $\begin{array}{l}\text { Vigna } \\
\text { unguiculata }\end{array}$ & $\begin{array}{l}\text { Zornia } \\
\text { capensis }\end{array}$ \\
\hline & $\mathrm{pH}(\mathrm{KCl})$ & $5.40 \pm 0.09^{\mathrm{ab}}$ & $5.48 \pm 0.11^{\mathrm{ab}}$ & $4.38 \pm 0.19^{b}$ & $4.85 \pm 0.34^{\mathrm{ab}}$ & $4.95 \pm 0.29^{\mathrm{ab}}$ & $5.35 \pm 0.12^{\mathrm{ab}}$ & $5.38 \pm 0.11^{\mathrm{ab}}$ & $5.41 \pm 0.17^{\mathrm{ab}}$ & $5.58 \pm 0.12^{\mathrm{ab}}$ & $5.87 \pm 0.58^{\mathrm{a}}$ & $5.52 \pm 0.34^{\mathrm{ab}}$ \\
\hline & $\mathrm{NO}_{3}\left(\mathrm{mg} \mathrm{kg}^{-1}\right)$ & $8.26 \pm 0.05^{\mathrm{c}}$ & $8.68 \pm 0.10^{c}$ & $16.10 \pm 0.12^{\mathrm{b}}$ & $6.45 \pm 0.12^{\mathrm{d}}$ & $0.29 \pm 0.03^{\mathrm{h}}$ & $3.77 \pm 0.12^{\mathrm{f}}$ & $5.14 \pm 0.07^{\mathrm{e}}$ & $28.40 \pm 0.42^{\mathrm{a}}$ & $8.51 \pm 0.09^{c}$ & $8.48 \pm 0.26^{\mathrm{c}}$ & $1.46 \pm 0.06^{\mathrm{g}}$ \\
\hline & $\mathrm{NH}_{4}\left(\mathrm{mg} \mathrm{kg}^{-1}\right)$ & $1.35 \pm 0.02^{\mathrm{f}}$ & $1.96 \pm 0.02^{\mathrm{d}}$ & $5.79 \pm 0.11^{\mathrm{a}}$ & $1.16 \pm 0.02^{\mathrm{fg}}$ & $0.96 \pm 0.02^{\mathrm{g}}$ & $3.61 \pm 0.09^{\mathrm{b}}$ & $1.73 \pm 0.01^{\mathrm{e}}$ & $2.65 \pm 0.02^{c}$ & $2.53 \pm 0.03^{c}$ & $2.18 \pm 0.01^{\mathrm{d}}$ & $3.64 \pm 0.01^{\mathrm{b}}$ \\
\hline & $\mathrm{P}\left(\mathrm{mg} \mathrm{kg}^{-1}\right)$ & $1.14 \pm 0.03^{\mathrm{gh}}$ & $3.33 \pm 0.03^{\mathrm{e}}$ & $4.77 \pm 0.02^{\mathrm{d}}$ & $2.46 \pm 0.06^{\mathrm{f}}$ & $1.24 \pm 0.03^{\text {gh }}$ & $7.12 \pm 0.02^{\mathrm{b}}$ & $1.53 \pm 0.24^{\mathrm{g}}$ & $5.44 \pm 0.04^{\mathrm{c}}$ & $1.02 \pm 0.02^{\mathrm{h}}$ & $9.30 \pm 0.14^{\mathrm{a}}$ & $2.07 \pm 0.01^{\mathrm{f}}$ \\
\hline & Organic $\mathrm{C}(\%)$ & $0.96 \pm 0.02^{\mathrm{h}}$ & $1.00 \pm 0.01^{\mathrm{h}}$ & $3.26 \pm 0.01^{\mathrm{c}}$ & $1.50 \pm 0.01^{\mathrm{g}}$ & $2.27 \pm 0.01^{\mathrm{e}}$ & $2.66 \pm 0.01^{\mathrm{d}}$ & $3.45 \pm 0.01^{\mathrm{b}}$ & $0.73 \pm 0.01^{\mathrm{i}}$ & $4.04 \pm 0.04^{\mathrm{a}}$ & $0.65 \pm 0.02^{\mathrm{j}}$ & $2.00 \pm 0.01^{\mathrm{f}}$ \\
\hline & $\mathrm{K}\left(\mathrm{mg} \mathrm{kg}^{-1}\right)$ & $94.40 \pm 0.25^{\mathrm{h}}$ & $130.00 \pm 0.02^{\mathrm{f}}$ & $175.00 \pm 0.41^{\mathrm{d}}$ & $224.00 \pm 3.21^{\mathrm{c}}$ & $188.00 \pm 0.26^{\mathrm{d}}$ & $233.00 \pm 0.13^{c}$ & $282.00 \pm 7.24^{b}$ & $155.00 \pm 5.77^{\mathrm{e}}$ & $394.00 \pm 3.25^{\mathrm{a}}$ & $113.00 \pm 0.12^{\mathrm{g}}$ & $153.00 \pm 0.05^{\mathrm{e}}$ \\
\hline & $\mathrm{Cu}\left(\mathrm{mg} \mathrm{kg}^{-1}\right)$ & $3.37 \pm 0.01^{\mathrm{a}}$ & $0.97 \pm 0.01^{\mathrm{g}}$ & $1.18 \pm 0.04^{\mathrm{f}}$ & $0.25 \pm 0.02^{\mathrm{i}}$ & $1.75 \pm 0.02^{\mathrm{e}}$ & $0.77 \pm 0.03^{\mathrm{h}}$ & $1.85 \pm 0.01^{\mathrm{d}}$ & $2.06 \pm 0.02^{c}$ & $2.26 \pm 0.02^{\mathrm{b}}$ & $0.81 \pm 0.02^{\mathrm{h}}$ & $1.15 \pm 0.02^{\mathrm{f}}$ \\
\hline & $\mathrm{Mn}\left(\mathrm{mg} \mathrm{kg}^{-1}\right)$ & $84.40 \pm 0.25^{\mathrm{a}}$ & $40.10 \pm 0.07^{\mathrm{e}}$ & $35.60 \pm 0.63^{\mathrm{h}}$ & $11.40 \pm 0.03^{\mathrm{j}}$ & $46.30 \pm 0.35^{c}$ & $36.50 \pm 0.30^{\mathrm{gh}}$ & $42.30 \pm 0.06^{\mathrm{d}}$ & $37.80 \pm 0.10^{\mathrm{f}}$ & $52.50 \pm 0.28^{\mathrm{b}}$ & $27.20 \pm 0.05^{\mathrm{i}}$ & $37.80 \pm 0.07^{\mathrm{fg}}$ \\
\hline & $\mathrm{Zn}\left(\mathrm{mg} \mathrm{kg}^{-1}\right)$ & $4.72 \pm 0.03^{\mathrm{e}}$ & $3.93 \pm 0.02^{\mathrm{f}}$ & $8.58 \pm 0.02^{\mathrm{c}}$ & $0.76 \pm 0.01^{\mathrm{i}}$ & $1.95 \pm 0.02^{\mathrm{h}}$ & $12.30 \pm 0.05^{\mathrm{b}}$ & $4.05 \pm 0.02^{\mathrm{f}}$ & $7.44 \pm 0.03^{\mathrm{d}}$ & $12.00 \pm 0.03^{\mathrm{b}}$ & $3.45 \pm 0.02^{\mathrm{g}}$ & $14.50 \pm 0.26^{\mathrm{a}}$ \\
\hline & $\mathrm{BD}\left(\mathrm{gcm}^{-3}\right)$ & $1.45 \pm 0.05^{\mathrm{ns}}$ & $1.55 \pm 0.04^{\mathrm{ns}}$ & $1.53 \pm 0.0$ & $1.51 \pm 0.02^{\mathrm{ns}}$ & $1.50 \pm 0.03^{\mathrm{ns}}$ & $1.59 \pm 0.09^{\mathrm{ns}}$ & $1.59 \pm 0.08^{\mathrm{ns}}$ & $1.45 \pm 0.12^{\mathrm{ns}}$ & $1.53 \pm 0.10^{\mathrm{ns}}$ & $1.51 \pm 0.01^{\mathrm{ns}}$ & \\
\hline & Sand $(\%)$ & $74.00 \pm 3.06^{\mathrm{ac}}$ & $76.00 \pm 3.00^{\mathrm{ac}}$ & $40.00 \pm 3.61^{\mathrm{d}}$ & $73.00 \pm 0.44^{\mathrm{ac}}$ & $78.00 \pm 1.25^{\mathrm{a}}$ & $74.00 \pm 1.53^{\mathrm{ac}}$ & $65.00 \pm 2.52^{c}$ & $38.00 \pm 1.73^{\mathrm{d}}$ & $74.00 \pm 1.15^{\mathrm{ac}}$ & $66.00 \pm 1.73^{\text {bc }}$ & $77.00 \pm 1.83^{\mathrm{ab}}$ \\
\hline & Silt (\%) & $14.00 \pm 1.15^{\mathrm{ab}}$ & $10.00 \pm 1.15^{\mathrm{b}}$ & $12.00 \pm 1.53^{\mathrm{ab}}$ & $15.00 \pm 1.53^{\mathrm{ab}}$ & $8.00 \pm 1.53^{\mathrm{b}}$ & $13.00 \pm 1.53^{\mathrm{b}}$ & $20.00 \pm 2.65^{\mathrm{a}}$ & $12.00 \pm 2^{\mathrm{ab}}$ & $14.00 \pm 2.08^{\mathrm{ab}}$ & $13.00 \pm 2.00^{\mathrm{ab}}$ & $10.00 \pm 1.53^{\mathrm{b}}$ \\
\hline & Clay $(\%)$ & $12.00 \pm 1.53^{\mathrm{b}}$ & $14.00 \pm 1.53^{\mathrm{b}}$ & $48.00 \pm 4.16^{\mathrm{a}}$ & $12.00 \pm 1.15^{\mathrm{b}}$ & $14.00 \pm 3.06^{\mathrm{b}}$ & $13.00 \pm 1.15^{\mathrm{b}}$ & $15.00 \pm 2.52^{\mathrm{b}}$ & $50.00 \pm 2.89^{\mathrm{a}}$ & $12.00 \pm 2.65^{\mathrm{b}}$ & $21.00 \pm 3^{\mathrm{b}}$ & $13.00 \pm 1.53^{\mathrm{b}}$ \\
\hline & Textural Class & SaLm & SaLm & $\mathrm{Cl}$ & $\mathrm{SaLm}$ & $\mathrm{SaLm}$ & $\mathrm{SaLm}$ & $\mathrm{SaLm}$ & $\mathrm{SaCl}$ & $\mathrm{SaLm}$ & SaClLm & $\mathrm{SaLm}$ \\
\hline \multirow[t]{14}{*}{ Mpumalanga } & $\mathrm{pH}(\mathrm{KCl})$ & $5.84 \pm 0.20^{\mathrm{a}}$ & $5.61 \pm 0.10^{\mathrm{ab}}$ & & $3^{\text {ac }}$ & $5.39 \pm 0.17^{\mathrm{ac}}$ & $4.64 \pm 0.28^{\mathrm{bc}}$ & $5.63 \pm 0.23^{\mathrm{ab}}$ & & $4.61 \pm 0.05^{\mathrm{bc}}$ & & \\
\hline & $\mathrm{NO}_{3}\left(\mathrm{mg} \mathrm{kg}^{-1}\right)$ & $4.31 \pm 0.13^{\mathrm{g}}$ & $6.28 \pm 0.16^{\mathrm{e}}$ & $11.10 \pm 0.08^{c}$ & $10.90 \pm 0.05^{\mathrm{c}}$ & $5.37 \pm 0.05^{\mathrm{f}}$ & $8.99 \pm 0.16^{\mathrm{d}}$ & $3.22 \pm 0.06^{\mathrm{h}}$ & $19.50 \pm 0.28^{\mathrm{a}}$ & $3.68 \pm 0.07^{\text {gh }}$ & $13.90 \pm 0.18^{\mathrm{b}}$ & $0.60 \pm 0.13^{\mathrm{i}}$ \\
\hline & $\mathrm{NH}_{4}\left(\mathrm{mg} \mathrm{kg}^{-1}\right)$ & $3.46 \pm 0.01^{\mathrm{b}}$ & $2.60 \pm 0.03^{\mathrm{c}}$ & $17.80 \pm 0.13^{\mathrm{a}}$ & $3.47 \pm 0.01^{\mathrm{b}}$ & $1.28 \pm 0.01^{\mathrm{ef}}$ & $2.73 \pm 0.10^{c}$ & $1.50 \pm 0.01^{\mathrm{de}}$ & $1.23 \pm 0.01^{\mathrm{f}}$ & $1.42 \pm 0.01^{\mathrm{df}}$ & $1.25 \pm 0.02^{\mathrm{f}}$ & $1.67 \pm 0.01^{\mathrm{d}}$ \\
\hline & $\mathrm{P}\left(\mathrm{mg} \mathrm{kg}^{-1}\right)$ & $3.06 \pm 0.02^{\mathrm{f}}$ & $4.45 \pm 0.02^{\mathrm{e}}$ & $2.02 \pm 0.02^{\mathrm{g}}$ & $1.04 \pm 0.03^{\mathrm{h}}$ & $5.21 \pm 0.04^{\mathrm{d}}$ & $11.20 \pm 0.03^{\mathrm{a}}$ & $2.33 \pm 0.06^{\mathrm{g}}$ & $8.64 \pm 0.12^{\mathrm{b}}$ & $2.14 \pm 0.05^{\mathrm{g}}$ & $6.20 \pm 0.18^{c}$ & $3.04 \pm 0.01^{\mathrm{f}}$ \\
\hline & Organic C $(\%)$ & $1.55 \pm 0.02^{\mathrm{e}}$ & $2.59 \pm 0.03^{c}$ & $5.34 \pm 0.02^{\mathrm{a}}$ & $5.35 \pm 0.02^{\mathrm{a}}$ & $1.54 \pm 0.02^{\mathrm{e}}$ & $4.77 \pm 0.01^{\mathrm{b}}$ & $1.89 \pm 0.01^{\mathrm{d}}$ & $1.57 \pm 0.01^{\mathrm{e}}$ & $1.00 \pm 0.03^{\mathrm{g}}$ & $1.96 \pm 0.05^{\mathrm{d}}$ & $1.36 \pm 0.01^{\mathrm{f}}$ \\
\hline & $\mathrm{K}\left(\mathrm{mg} \mathrm{kg}^{-1}\right)$ & $131.00 \pm 0.42^{\mathrm{e}}$ & $163.00 \pm 0.10^{\mathrm{d}}$ & $233.00 \pm 0.06^{\mathrm{ac}}$ & $247 \pm 3.31^{\mathrm{ab}}$ & $135.00 \pm 14.60^{\mathrm{e}}$ & $163.00 \pm 0.05^{\mathrm{d}}$ & $223.00 \pm 2.81^{\mathrm{bc}}$ & $218.00 \pm 8.77^{c}$ & $258.00 \pm 3.43^{\mathrm{a}}$ & $103.00 \pm 0.52^{\mathrm{f}}$ & $138.00 \pm 0.06^{\mathrm{de}}$ \\
\hline & $\mathrm{Cu}\left(\mathrm{mg} \mathrm{kg}^{-1}\right)$ & $0.28 \pm 0.01^{\mathrm{h}}$ & $1.77 \pm 0.01^{\mathrm{e}}$ & $3.73 \pm 0.03^{\mathrm{b}}$ & $1.06 \pm 0.03^{\mathrm{g}}$ & $11.60 \pm 0.03^{\mathrm{a}}$ & $3.18 \pm 0.03^{\mathrm{c}}$ & $1.57 \pm 0.01^{\mathrm{f}}$ & $0.21 \pm 0.02^{\mathrm{h}}$ & $0.24 \pm 0.02^{\mathrm{h}}$ & $2.44 \pm 0.07^{\mathrm{d}}$ & $1.03 \pm 0.04^{\mathrm{g}}$ \\
\hline & $\mathrm{Mn}\left(\mathrm{mg} \mathrm{kg}^{-1}\right)$ & $25.40 \pm 0.03^{\mathrm{e}}$ & $53.00 \pm 0.06^{\mathrm{c}}$ & $84.40 \pm 0.12^{b}$ & $131.00 \pm 0.42^{a}$ & $25.60 \pm 0.03^{\mathrm{e}}$ & $29.40 \pm 0.05^{\mathrm{d}}$ & $25.40 \pm 0.06^{\mathrm{e}}$ & $1.08 \pm 0.11^{\mathrm{i}}$ & $13.10 \pm 0.06^{\mathrm{h}}$ & $16.10 \pm 0.02^{\mathrm{g}}$ & $18.10 \pm 0.09^{\mathrm{f}}$ \\
\hline & $\mathrm{Zn}\left(\mathrm{mg} \mathrm{kg}^{-1}\right)$ & $2.09 \pm 0.04^{\mathrm{e}}$ & $6.11 \pm 0.04^{c}$ & $13.40 \pm 0.05^{\mathrm{a}}$ & $6.15 \pm 0.02^{\mathrm{bc}}$ & $1.34 \pm 0.01^{\mathrm{g}}$ & $3.68 \pm 0.02^{\mathrm{d}}$ & $6.26 \pm 0.02^{b}$ & $0.67 \pm 0.01^{\mathrm{i}}$ & $1.07 \pm 0.01^{\mathrm{h}}$ & $1.48 \pm 0.01^{\mathrm{f}}$ & $1.46 \pm 0.01^{\mathrm{fg}}$ \\
\hline & $\mathrm{BD}\left(\mathrm{gcm}^{-3}\right)$ & $1.49 \pm 0.12^{\mathrm{ns}}$ & $1.51 \pm 0.03^{\mathrm{ns}}$ & $1.59 \pm 0.04^{\mathrm{ns}}$ & $1.55 \pm 0.02^{\mathrm{ns}}$ & $1.58 \pm 0.06^{\mathrm{ns}}$ & $1.60 \pm 0.03^{\mathrm{ns}}$ & $1.48 \pm 0.05^{\mathrm{ns}}$ & $1.54 \pm 0.07^{\mathrm{ns}}$ & $1.57 \pm 0.09^{\mathrm{ns}}$ & $1.53 \pm 0.06^{\mathrm{ns}}$ & $1.60 \pm 0.08^{\mathrm{ns}}$ \\
\hline & Sand $(\%)$ & $75.00 \pm 0.84^{\mathrm{ab}}$ & $75.00 \pm 1.73^{\mathrm{ab}}$ & $52.00 \pm 1.73^{c}$ & $68.00 \pm 0.49^{\mathrm{b}}$ & $67.00 \pm 2.30^{\mathrm{b}}$ & $36.00 \pm 1.53^{\mathrm{d}}$ & $68.00 \pm 3.32^{\mathrm{b}}$ & $50.00 \pm 2.89^{c}$ & $83.00 \pm 2.31^{\mathrm{a}}$ & $34.00 \pm 2.39^{d}$ & $33.00 \pm 1.33^{\mathrm{d}}$ \\
\hline & Silt (\%) & $11.00 \pm 1.15^{\mathrm{cd}}$ & $13.00 \pm 2.52^{\mathrm{cd}}$ & $10.00 \pm 1.53^{\text {cd }}$ & $14.00 \pm 2.31^{\mathrm{cd}}$ & $10.00 \pm 2.00^{\mathrm{cd}}$ & $42.00 \pm 3.06^{\mathrm{a}}$ & $20.00 \pm 4.62^{\mathrm{bc}}$ & $8.00 \pm 1.53^{\mathrm{cd}}$ & $6.00 \pm 1.15^{\mathrm{d}}$ & $27.00 \pm 3.61^{\mathrm{b}}$ & $19.00 \pm 2.00^{\mathrm{bc}}$ \\
\hline & Clay (\%) & $14.00 \pm 2.00^{\mathrm{b}}$ & $12.00 \pm 1.15^{\mathrm{b}}$ & $38.00 \pm 3.00^{\mathrm{a}}$ & $18.00 \pm 2.08^{\mathrm{b}}$ & $23.00 \pm 1.73^{\mathrm{b}}$ & $22.00 \pm 2.31^{\mathrm{b}}$ & $12.00 \pm 2.00^{\mathrm{b}}$ & $42.00 \pm 1.15^{\mathrm{a}}$ & $11.00 \pm 1.53^{\mathrm{b}}$ & $39.00 \pm 4.04^{\mathrm{a}}$ & $48.00 \pm 4.16^{\mathrm{a}}$ \\
\hline & Textural Class & $\mathrm{SaLm}$ & SaLm & $\mathrm{SaCl}$ & SaLm & SaClLm & $\mathrm{Lm}$ & $\mathrm{SaLm}$ & $\mathrm{Cl}$ & $\mathrm{LmSa}$ & ClLm & $\mathrm{Cl}$ \\
\hline
\end{tabular}

Note: Values are given as means \pm SEM. Means across rows and columns without a common superscript alphabet letters statistically differ $(P<0.05)$ according to two-way ANOVA and the Loam; Cl, Clay; SaCl, Sandy Clay; SaClLm, Sandy Clay Loam; Lm, Loam; ClLm, Clay Loam; LmSa; Loamy Sand. 
Table 2. Spore density and diversity indices of AMF in different legumes in both provinces

\begin{tabular}{|c|c|c|c|c|c|c|c|c|c|c|c|c|}
\hline Provinces & Indices & C. comosa & C. distans & E. cordatum & I. evansiana & R. minima & R. venulosa & T. africanum & T. kraussiana & T. repens & V. unguiculata & Z. capensis \\
\hline \multirow[t]{4}{*}{ Gauteng } & SD & $541.00 \pm 42.10^{\mathrm{a}}$ & $554.00 \pm 43.30^{\mathrm{a}}$ & $384.00 \pm 26.35^{\text {ba }}$ & $519.00 \pm 25.20^{\text {aа }}$ & $478.00 \pm 35.30^{\text {ba }}$ & $426.00 \pm 34.90^{\mathrm{ba}}$ & $786.00 \pm 29.24^{\mathrm{c}}$ & $306.00 \pm 35.50^{d}$ & $812.00 \pm 45.60^{\mathrm{c}}$ & $433.00 \pm 33.30^{\text {ba }}$ & $569.00 \pm 53.10^{\mathrm{a}}$ \\
\hline & SR & $8.00 \pm 1.31^{\mathrm{a}}$ & $9.00 \pm 1.15^{\mathrm{a}}$ & $6.00 \pm 1.35^{\mathrm{ac}}$ & $11.00 \pm 1.73^{\mathrm{a}}$ & $5.00 \pm 1.43^{\mathrm{ac}}$ & $8.00 \pm 2.31^{\mathrm{a}}$ & $12.00 \pm 1.05^{\mathrm{b}}$ & $4.00 \pm 0.98^{a c}$ & $18.00 \pm 2.89^{\mathrm{b}}$ & $7.00 \pm 1.55^{\mathrm{a}}$ & $8.00 \pm$ \\
\hline & $\mathrm{H}$ & $1.82 \pm 0.59^{\mathrm{a}}$ & $2.06 \pm 0.54^{\mathrm{a}}$ & $1.70 \pm 0.52^{\mathrm{a}}$ & $2.33 \pm 0.30^{\mathrm{a}}$ & $1.57 \pm 0.55^{\mathrm{ab}}$ & $1.99 \pm 0.58^{\mathrm{a}}$ & $2.32 \pm 0.20^{\mathrm{ac}}$ & $1.38 \pm 0.54^{\mathrm{b}}$ & $2.72 \pm 1.10^{c}$ & $1.89 \pm 0.57^{\mathrm{a}}$ & $2.06 \pm 0.27^{\mathrm{a}}$ \\
\hline & $\mathrm{J}$ & $0.87 \pm 0.08^{\mathrm{ns}}$ & $0.94 \pm 0.05^{\mathrm{ns}}$ & $0.95 \pm 0.03^{\mathrm{ns}}$ & $0.97 \pm 0.01^{\mathrm{ns}}$ & $0.98 \pm 0.01^{\mathrm{ns}}$ & $0.98 \pm 0.02^{\mathrm{ns}}$ & $0.93 \pm 0.02^{\mathrm{ns}}$ & $0.99 \pm 0.01^{\mathrm{ns}}$ & $0.94 \pm 0.03^{\mathrm{ns}}$ & $0.97 \pm 0.02^{\mathrm{ns}}$ & $0.99 \pm 0.06^{\mathrm{ns}}$ \\
\hline \multirow[t]{4}{*}{ Mpumalanga } & SD & $508.00 \pm 33.84^{\mathrm{a}}$ & $581.00 \pm 30.00^{\mathrm{a}}$ & $419.00 \pm 24.00^{\mathrm{ba}}$ & $557.00 \pm 28.30^{\mathrm{ba}}$ & $459.00 \pm 23.38^{\text {ba }}$ & $460.00 \pm 23.60^{\mathrm{ba}}$ & $738.00 \pm 37.80^{c}$ & $284.00 \pm 26.20^{\mathrm{d}}$ & $34.30^{c}$ & $389.00 \pm 53.30^{\text {ba }}$ & \\
\hline & SR & $6.00 \pm 1.53^{\mathrm{a}}$ & $6.00 \pm 0.58^{\mathrm{a}}$ & $4.00 \pm 0.76^{\mathrm{a}}$ & $7.00 \pm 1.15^{\mathrm{a}}$ & $4.00 \pm 0.53^{\mathrm{a}}$ & $8.00 \pm 2.31^{\mathrm{a}}$ & $15.00 \pm 1.27^{\mathrm{b}}$ & $5.00 \pm 0.58^{\mathrm{a}}$ & $14.00 \pm 2.31^{\mathrm{b}}$ & $9.00 \pm 0.99^{\mathrm{a}}$ & $10.00 \pm 2.08^{\mathrm{a}}$ \\
\hline & $\mathrm{H}$ & $1.75 \pm 0.44^{\mathrm{a}}$ & $1.76 \pm 0.56^{\mathrm{a}}$ & $1.37 \pm 0.57^{\mathrm{ab}}$ & $1.93 \pm 0.58^{\mathrm{a}}$ & $1.31 \pm 0.49^{\mathrm{ab}}$ & $2.02 \pm 0.56^{\mathrm{a}}$ & $2.61 \pm 0.18^{c}$ & $1.54 \pm 0.55^{\mathrm{ab}}$ & $2.50 \pm 0.29^{c}$ & $2.20 \pm 0.44^{\mathrm{a}}$ & $2.20 \pm 0.42^{\mathrm{a}}$ \\
\hline & $\mathrm{J}$ & $0.98 \pm 0.05^{\mathrm{ns}}$ & $0.98 \pm 0.09^{\text {ns }}$ & $0.99 \pm 0.04^{\mathrm{ns}}$ & $0.99 \pm 0.02^{\mathrm{ns}}$ & $0.95 \pm 0.02^{\mathrm{ns}}$ & $0.97 \pm 0.01^{\mathrm{ns}}$ & $0.96 \pm 0.01^{\mathrm{ns}}$ & $0.96 \pm 0.03^{\mathrm{ns}}$ & $0.95 \pm 0.01^{\mathrm{ns}}$ & $0.96 \pm 0.09^{\mathrm{ns}}$ & $0.95 \pm 0.01^{\mathrm{ns}}$ \\
\hline
\end{tabular}

Note: Values are means \pm SEM. Means across rows and columns without a common superscript alphabet letters statistically differ $(P<0.05)$ based on two-way ANOVA and the TUKEY test. ns, not significant; SR, H, and J are the species richness, Shannon-Weiner diversity index, and Pielou evenness index, respectively. 


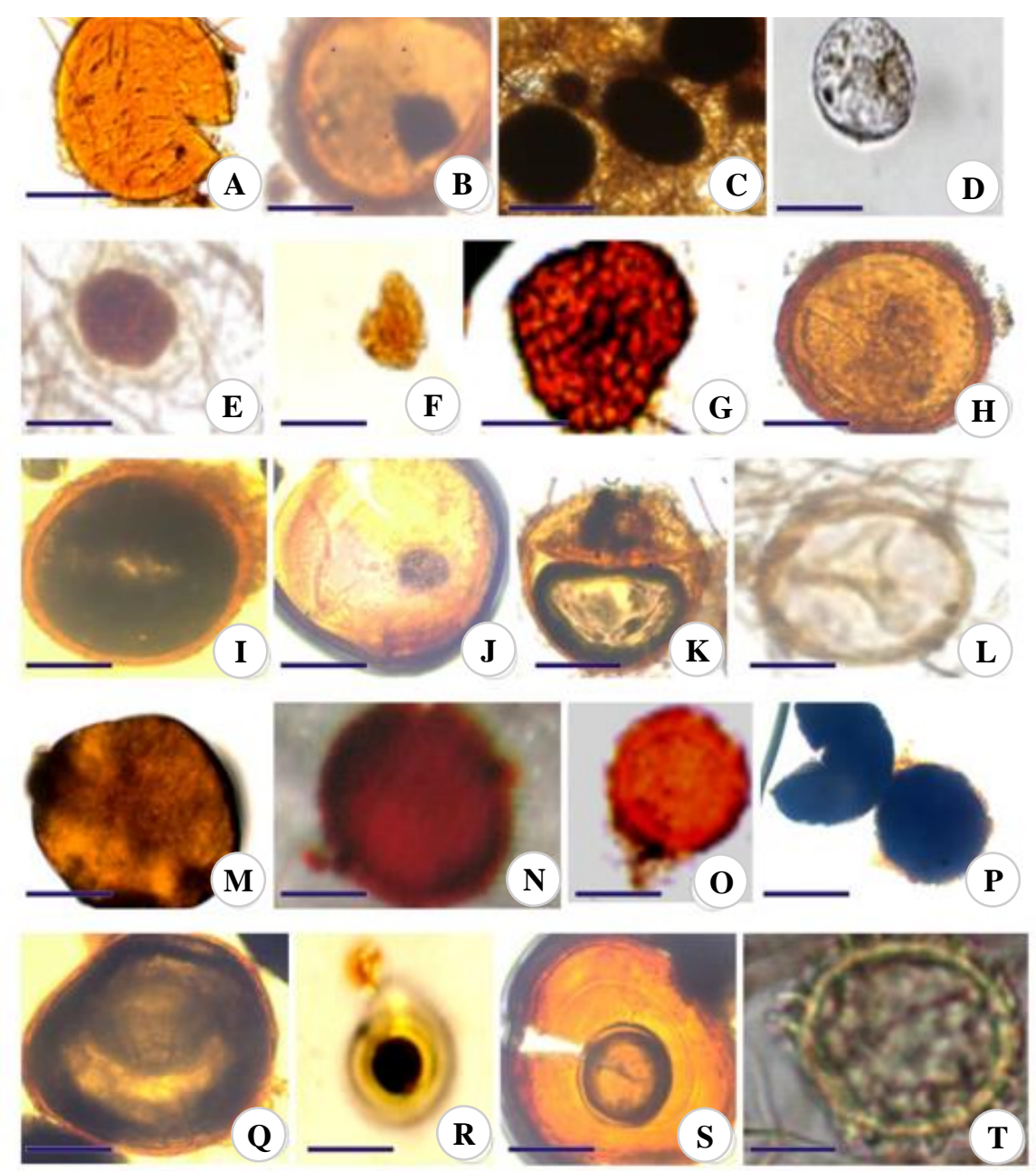

Figure 2. Diversity of AM fungal species identified in the rhizosphere soils of legumes in both provinces. A. Glomus magnicaule B. Glomus delhiense C. Glomus ambisporum D. Glomus tubaeforme E. Glomus rubiforme F. Glomus sinuosum G. Glomus taiwanense H. Acaulospora colombiana I. Acaulospora mellea J. Acaulospora tuberculata K. Rhizophagus intraradices L. Rhizophagus irregularis M. Septoglomus deserticola N. Septoglomus constrictum O. Claroideoglomus etunicatum P. Entrophospora infrequens Q. Funneliformis geosporum R. Gigaspora decipiens S. Scutellospora biornata T. Sieverdingia tortuosa. All scale bars $=50 \mu \mathrm{m}$ except; B $=35 \mu \mathrm{m}$ and G $=30 \mu \mathrm{m}$.

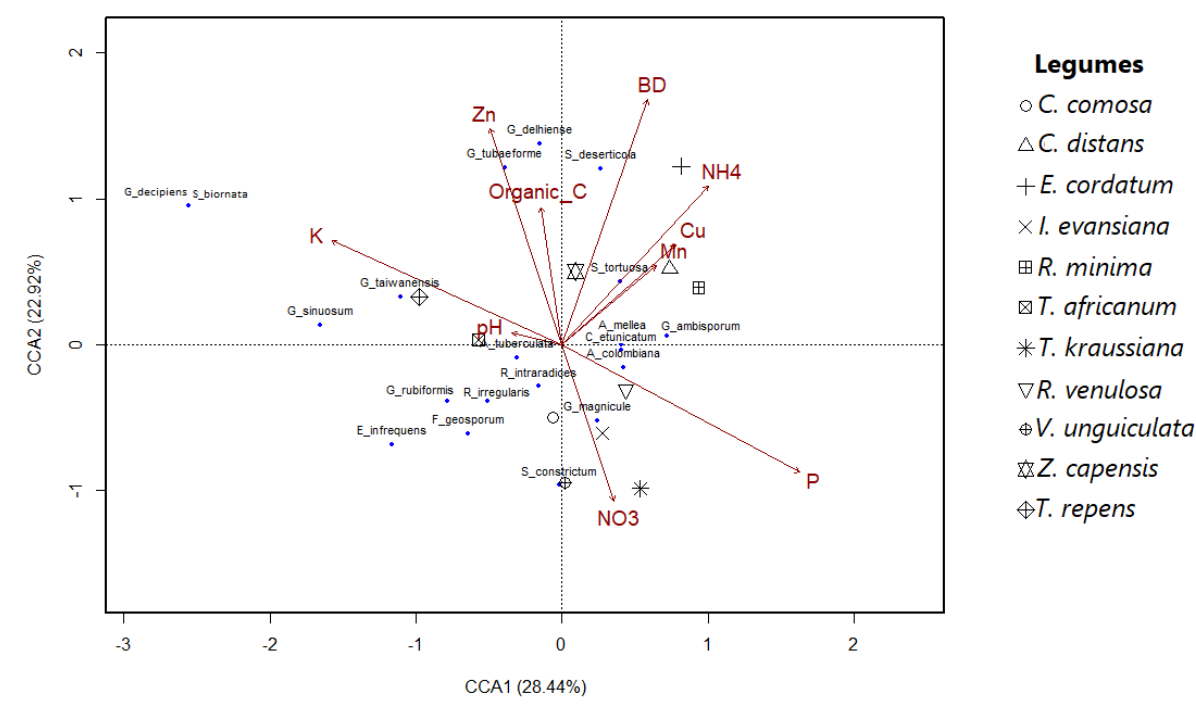

Figure 3. The canonical correspondence analysis (CCA) showing the relationship between AMF communities and soil properties. $\mathrm{NO}_{3}$, nitrate; $\mathrm{NH}_{4}$, ammonia; $\mathrm{P}$, available phosphorus; $\mathrm{K}$, available potassium; $\mathrm{Cu}$, copper; $\mathrm{Mn}$, manganese; $\mathrm{Zn}$, zinc; $\mathrm{BD}$, Bulk density. 
Table 3. Correlation analysis between AM fungal spore density, diversity indices, and soil properties

\begin{tabular}{lllll}
\hline Parameters & SD & SR & H & J \\
\hline $\mathrm{pH}$ & $0.32^{*}$ & $0.35^{* *}$ & $0.44^{* * *}$ & $0.41^{* *}$ \\
$\mathrm{NO}_{3}$ & $-0.4^{* * *}$ & -0.23 & -0.13 & 0.10 \\
$\mathrm{NH}_{4}$ & -0.07 & -0.17 & -0.09 & 0.20 \\
$\mathrm{P}$ & $-0.59^{* * *}$ & $-0.30^{*}$ & -0.12 & 0.05 \\
Organic C & 0.13 & 0.05 & 0.01 & 0.07 \\
$\mathrm{~K}$ & $0.54^{* * *}$ & $0.49^{* * *}$ & 0.20 & 0.01 \\
$\mathrm{Cu}$ & -0.09 & -0.18 & -0.18 & -0.05 \\
$\mathrm{Mn}$ & $0.25^{*}$ & -0.14 & -0.13 & 0.12 \\
$\mathrm{Zn}$ & 0.11 & -0.01 & -0.02 & 0.14 \\
$\mathrm{BD}$ & 0.03 & $0.31^{*}$ & $0.5 * * *$ & $0.38^{* *}$ \\
$\mathrm{Sand}$ & 0.13 & -0.08 & -0.02 & 0.13 \\
Silt & -0.2 & -0.04 & 0.03 & 0.05 \\
$\mathrm{Clay}$ & 0.01 & 0.15 & 0.14 & 0.01 \\
\hline Not & & & 0.14
\end{tabular}

Note: $\mathrm{NO}_{3}$, nitrate; $\mathrm{NH} 4$, ammonia; $\mathrm{P}$, available phosphorus; $\mathrm{K}$, available potassium; $\mathrm{Cu}$, copper; $\mathrm{Mn}$, manganese; $\mathrm{Zn}$, zinc; $\mathrm{BD}$, Bulk density; SD, spore density; SR, spore richness; H, ShannonWeiner diversity index; J, Pielou evenness index; *Significant at $P<0.05$; **Significant at $P<0.01$; *** Significant at $P<0.001$.

Arbuscular mycorrhizal fungal spores possess key morphological features that enable species-level identification, and many studies have inferred the community structure of AMF based on spore communities (Singh et al. 2008; Wang et al. 2019). In this study, we reported for the first time, the diversity of AM fungal communities in the rhizosphere of eleven indigenous legumes of South Africa using spore morphology. A total of 20 AM fungal species were detected. This diversity is relatively higher than what has been previously reported in other host plants in this region (Gaur et al. 1999; Straker et al. 2010) and in different leguminous plants from other semi-arid ecosystems (Dalpé et al. 2000; Feitosa de Souza, 2016; Choosa-Nga et al. 2019).

Furthermore, the results showed that Glomus was the predominant genus, followed by Acaulospora. Previous researchers have reported the dominance of these genera in other legumes (Songachan and Kayang 2013; Choosa-Nga et al. 2019). The dominance of Glomus and Acaulospora may be attributed to their small spore morphology, ease of sporulation, and wider adaptability to different plants and environmental conditions (Hepper 1984). More so, the fact that these genera propagate mostly by spores, which are considered highly resistant propagules under harsh environmental conditions (Lennon and Jones 2011), could also support their dominance in the rhizosphere of examined plants.

The genera Gigaspora and Scutellospora were recorded in low numbers in this study. Species of the family Gigasporaceae are largely established through other propagules such as hyphae and mycelial fragments (Hart and Reader 2002). Moreover, studies have indicated that species of Gigasporaceae are more prevalent in sandy soils such as dunes (Lee and Koske 1994). Hence, the rarity of these genera may reflect their low competitive capacity and narrow adaptability to this environment. This study revealed that $A$. colombiana, A. mellea, and $C$. etunicatum were the most frequent AM fungal species. Acaulospora species have greater survival capacity in low soil $\mathrm{pH}$ since their occurrence is principally associated with acidic soils (Morton 1986; Straker et al. 2010). Claroideoglomus etunicatum is a cosmopolitan species in diverse ecotypes (Becker and Gerdemann 1977). Perhaps, the ability of these species to adapt well in vast environments is responsible for their commonality to all the legumes.

The AM fungal community structure varied in different legume species, which accords with the findings of Choosa-Nga et al. (2019) and Olubode et al. (2020). The dissimilarity may be owing to host preferences for different AM fungal species (Castillo et al. 2016; Wang et al. 2019), as well as the potential influence of soil properties (Sweeney et al. 2021). Preferential associations among host-AMF pairs have been attributed to the functional diversity among AM fungal species; whereby host plants filter and reward the most beneficial AM fungal partners in their local environments with more carbon (Bever et al. 2009; Kiers et al. 2011). The differential abundance of members of the families Glomeraceae and Acaulosporacea in the rhizosphere of different legumes may be an indication of their functional significance. Hence, this finding suggests the possibility of utilizing the abundant AM fungal species in the rhizosphere soils of respective legumes as inoculum for management purposes.

Soil environmental factors have been widely acknowledged as key environmental determinants of AMF diversity and community structure, particularly at local scales (Jansa et al. 2014; Alguacil et al. 2016). In this study, AM fungal SD and diversity indices were significantly correlated with soil $\mathrm{pH}$. $\mathrm{pH}$ is one of the most important environmental variables that shape AM fungal diversity and community structure by regulating the availability of nutrients and ions in the soil for plant uptake (Coughlan et al. 2000). Furthermore, since AM symbiosis is generally triggered by soil nutrient-limiting conditions; expectedly, AMF spore population and species richness should be inversely related to soil $\mathrm{P}$ and $\mathrm{N}$ levels (Read 1991). Thus, the lower $P$ and $N$ concentrations in the rhizosphere soils of studied legumes may have contributed to the significant negative correlation observed in this study.

Excessive soil compaction adversely affects plant root growth and AMF sporulation (Nadian et al. 1998; Yano et al. 1998). Soil bulk density is one of the most frequently used measures of compaction. Although the critical value of bulk density for inhibiting root growth differs with soil type, bulk density values greater than $1.6 \mathrm{~g} / \mathrm{cm}^{3}$ appear to restrict root growth and affect AMF activities (McKenzie et al. 2004). The values of bulk density recorded in the rhizosphere of legumes in this study fall within this range, and hence may account for the positive relationship observed between diversity indices, AM fungal communities, and BD. Significant positive association was also recorded between SD, SR, AM fungal communities and soil $\mathrm{Mn}$ and Zinc contents. These micronutrients play important roles in legume metabolic process, most 
especially in nitrogen fixation and their uptake can be positively impacted by the presence of AMF (Weisany et al. 2013; Lehmann and Rillig 2015).

The stimulatory effects of soil $\mathrm{K}$ on AM fungal attributes have been reported (Furlan et al. 1989). The high soil $\mathrm{K}$ concentration recorded in the rhizosphere of studied legumes could have promoted AMF spore production, and in turn, enhanced AMF species richness and community structure. While plant-AMF symbiosis is in response to soil nutrient limitations, variation in the structure of rhizosphere AMF communities may result from complex interactions between biotic and abiotic factors (Dumbrell et al. 2010). The distribution of $R$. intraradices, $G$. rubiforme, $R$. irregularis, E. infrequens, $F$. geosporum, and $S$. constrictum were least affected by soil properties, thereby implying the role of other factors (presumably host plants, climate, or dispersal capabilities of AM fungal species) in shaping the structure of these species.

In conclusion, this study reveals the dominance and abundance of Glomeraceae and Acaulosporaceae in the rhizosphere of the examined legumes. This suggests that genera of both families may be particularly important in the establishment and adaptation of legumes to this environment. Accordingly, they could be the most promising genera for use as inoculants to further investigate the ecological impacts of the symbiotic interactions between AMF and legumes. Spore density and species richness were highest in Trifolium species, indicating their rhizosphere soils as a great source of inoculum. Overall, this study provides a valuable contribution to the biodiversity of AMF present in semiarid soils and demonstrated the effect of soil environmental factors on AMF diversity and community structure.

\section{ACKNOWLEDGEMENTS}

This work is based on research supported in part by the National Research Foundation of South Africa for Grant number 76177. The University of Johannesburg is acknowledged for financial and logistical assistance. The authors are thankful to the Taxonomy and Ethnobotany (TAXETH) research group for assisting during fieldwork, as well as Dr. O.T. Ezeokoli and T. Akinosho for their help with statistical analyses.

\section{REFERENCES}

Addy HD, Schaffer GF, Miller MH, Peterson RL. 1994. Survival of the external mycelium of a VAM fungus in frozen soil over winter. Mycorrhiza 5 (1): 1-5. DOI: 10.1007/BF00204013.

Alguacil MDM, Torres MP, Montesinos-Navarro A, Roldán A. 2016. Soil characteristics driving arbuscular mycorrhizal fungi communities in semiarid Mediterranean soils. Appl Environ Microbiol 82: 33483356. DOI: 10.1016/j.funeco.2019.02.002.

Anderson RC, Liberta AE, Dickman LA. 1984. Interaction of vascular plants and vesicular-arbuscular mycorrhizal fungi across a soil moisture-nutrient gradient. Oecologia 64 (1): 111-117. DOI 10.1007/BF00377552.

Banwart WL, Tabatabai MA, Bremner BJ. 1972. Determination of ammonium in soil extracts and water samples by an ammonia electrode 1. Commun Soil Sci Plant Anal 3: 449-458. DOI: 10.1080/00103627209366401.

Barea JM, Azcón-Aguilar C, Azcón R.1987. Vesicular-arbuscular mycorrhiza improve both symbiotic $\mathrm{N}_{2}$ fixation and $\mathrm{N}$ uptake from soil as assessed with a ${ }^{15} \mathrm{~N}$ technique under field conditions. New Phytol 106 (4): 717-725. DOI: 10.1111/j.1469-8137.1987.tb00172.x.

Barnard RO, du Preez CC. 2004. Soil fertility in South Africa: the last twenty-five years. S Afr J Plant Soil 21 (5): 301-315. DOI: 10.1080/02571862.2004.10635066.

Becker WN, Gerdemann JW. 1977. Glomus etunicatus sp. nov. Mycotaxon 6: 29-32.

Bever JD, Richardson SC, Lawrence BM, Holmes J, Watson M. 2009. Preferential allocation to beneficial symbiont with spatial structure maintains mycorrhizal mutualism. Ecol Lett 12 (1): 13-21. DOI: 10.1111/j.1461-0248.2008.01254.x.

Bouyoucos GJ. 1962. Hydrometer method improved for making particle size analyses of soils 1. Agron J 54: 464-465. DOI: 10.2134/agronj1962.00021962005400050028x.

Bray RH, Kurtz LT. 1945. Determination of total organic and available forms of phosphorus in soils. Soil Sci 59: 39-45. DOI: 10.1097/00010694-194501000-00006.

Brundrett M, Bougher N, Dell B, Grove T, Malajczuk N. 1996. Working with Mycorrhizas in Forestry and Agriculture. ACIAR Monograph 32. ACIAR, Australia.

Brundrett MC, Tedersoo L. 2018. Evolutionary history of mycorrhizal symbioses and global host plant diversity. New Phytol 220 (4): 11081115. DOI: $10.1111 / \mathrm{nph} .14976$.

Carrenho R, Trufem SFB, Bononi VLR. 2002. Effects of using different host plants in the detected biodiversity of arbuscular mycorrhizal fungi from an agroecosystem. Revista Brasileira de Botanica 25 (1): 93-101. DOI: 10.1590/S0100-84042002000100012.

Castillo CG, Borie F, Oehl F, Sieverding E. 2016. Arbuscular mycorrhizal fungi biodiversity: prospecting in Southern-Central zone of Chile. A review. J Soil Sci Plant Nutr 16 (2): 400-422. DOI: 10.4067/S071895162016005000036.

Chalk P, Souza R, Urquiaga S, Alves B, Boddey R. 2006. The role of arbuscular mycorrhiza in legume symbiotic performance. Soil Biol Biochem 38: 2944-2951. DOI: 10.1016/j.soilbio.2006.05.005.

Chen M. Arato M, Borghi L, Nouri E, Reinhardt D. 2018. Beneficial services of arbuscular mycorrhizal fungi - from ecology to application. Front Plant Sci 9: 1270. DOI: 10.3389/fpls.2018.01270.

Choosa-Nga P, Sangwanit U, Kaewgrajang T. 2019. The arbuscular mycorrhizal fungi's diversity in fabaceous trees species of Northeastern Thailand. Biodiversitas 20 (2): 405-412. DOI: 10.13057/biodiv/d200214.

Cleveland CC, Townsend AR, Schimel DS, Fisher H, Howarth RW, Hedin LO, Perakis SS, Latty EF, Von Fischer JC, Elseroad A, Wasson MF. 1999. Global patterns of terrestrial biological nitrogen $\left(\mathrm{N}_{2}\right)$ fixation in natural ecosystems. Global Biogeochem Cycles 13 (2): 623-645. DOI: 10.1029/1999GB900014.

Coughlan AP, Dalpé Y, Lapointe L, Piché Y. 2000. Soil pH-induced changes in root colonization, diversity, and reproduction of symbiotic arbuscular mycorrhizal fungi from healthy and declining maple forests. Can J For Res 30 (10): 1543-1554. DOI: 10.1139/x00-090.

D'arcy-Lameta A. 1988. Study of soybean and lentil root exudates. 11. Identification of some polyphenolic compounds related with plant physiology. Plant and Soil 92: 113-123. DOI: 10.1007/BF02370654.

Dalpé Y, Diop TA, Plenchette C, Gueye M. 2000. Glomales species associated with surface and deep rhizosphere of Faidherbia albida in Senegal. Mycorrhiza 10: 125-129. DOI: 10.1007/s005720000069.

Daniels BA, Skipper HD. 1982. Methods for the recovery and quantitative estimation of propagules from soil. In: Schenck NC. (ed). Methods and Principle of Mycorrhizal Research. American Phytopathological Society, USA.

de Oliveira JRG, de Resende GM, de Melo NF, Yano-Melo AM. 2017. Symbiotic compatibility between arbuscular mycorrhizal fungi (autochtone or exotic) and three native species of the Caatinga in different phosphorus levels. Acta Scientiarum Biological Sciences 39: 59-69. DOI: 10.4025/actascibiolsci.v39i1.33486.

Dumbrell AJ, Nelson M, Helgason T, Dytham C, Fitter AH. 2010. Relative roles of niche and neutral processes in structuring a soil microbial community. ISME J 4: 337-345. DOI: 10.1038/ismej.2009.122.

Eom AH, Hartnett DC, Wilson GWT. 2000. Host plant species effects on arbuscular mycorrhizal fungal communities in tallgrass prairie. Oecologia 122: 435-444. DOI: 10.1007/s004420050050. 
Feitosa de Souza TA, Rodriguez-Echeverría S, Alves de Andrade L, Freitas H. 2016. Arbuscular mycorrhizal fungi in Mimosa tenuiflora (Willd.) Poir from Brazilian semi-arid. Brazilian J Microbiol 47: 359366. DOI: 10.1016/j.bjm.2016.01.023.

Fitzsimons MS, Miller RM. 2010. The importance of soil microorganisms for maintaining diverse plant communities in tallgrass prairie. Am J Bioethics 97 (12): 1937-1943. DOI: 10.3732/ajb.0900237.

Furlan V, Bernier-Cardou M. 1989. Effect of N, P and K on formation of endomycorrhizae, growth and mineral content of onion. Plant and Soil 113: 167-174. DOI: 10.1007/BF02280177.

Gaur A, Van Greuning JV, Sinclair RC, Eicker A. 1999. Arbuscular mycorrhizas of Vangueria infausta Burch. subsp. Infausta (Rubiaceae) from South Africa. S Afr J Bot 65: 434-436. DOI: 10.1016/S0254-6299(15)31036-X.

Graham PH, Vance CP. 2003. Legumes: importance and constraints to greater use. Plant Physiol 131 (3): 872-877. DOI: 10.1104/pp.017004.

Hart MM, Reader RJ. 2002. Taxonomic basis for variation in the colonization strategy of arbuscular mycorrhizal fungi. New Phytol 153 (2): 335-344. DOI: 10.1046/j.0028-646X.2001.00312.x.

Hawkins HJ, Wolf G, Stock WD. 2005. Cluster roots of Leucadendron laureolum (Proteaceae) and Lupinus albus (Fabaceae) take up glycine intact: an adaptive strategy to low mineral nitrogen in soils? Ann Bot 96 (7): 1275-1282. DOI: $10.1093 / \mathrm{aob} / \mathrm{mci} 279$

Hawley GL, Dames JF. 2004. Mycorrhizal status of indigenous tree species in a forest biome of the Eastern Cape, South Africa. S Afr J Sci 100 (11-12): 633-637.

Hepper CM. 1984. Isolation and culture of VAM fungi. In: Powell CL, Bagyaraj DJ (eds). VA Mycorrhizae. CRC Press, Boca Raton, FL.

International Organization for Standardization, ISO. 2017. Soil qualitydetermination of dry bulk density. International Organization for Standardization, Geneva.

INVAM 1997. The international culture collection of arbuscular and VA mycorrhizal fungi (INVAM). httpss://www.invam.caf.wvu.edu/

Jackson ML. 1958. Soil Chemical Analysis. Prentice-Hall Inc., New York.

Jansa J, Erb A, Oberholzer H, Šmilauer P, Egli S. 2014. Soil and geography are more important determinants of indigenous arbuscular mycorrhizal communities than management practices in Swiss agricultural soils. Mol Ecol 23: 2118-2135. DOI: 10.1111/mec.12706.

Johnson D, Vandenkoornhuyse PJ, Leake JR, Gilbert L, Booth RE, Grime JP, Young JPW, Read DJ. 2004. Plant communities affect arbuscular mycorrhizal fungal diversity and community composition in grassland microcosms. New Phytol 161 (2): 503-515. DOI: 10.1046/j.14698137.2003.00938.x.

Jones DL, Hodge A, Kuzyakov Y. 2004. Plant and mycorrhizal regulation of rhizodeposition. New Phytol 163: 459-80. DOI: 10.1111/j.1469 8137.2004.01130.x.

Kiers ET, Duhamel M, Beesetty Y, Mensah JA, Franken O, Verbruggen E, Fellbaum CR, Kowalchuk GA, Hart MM, Bago A, Palmer TM 2011. Reciprocal rewards stabilize cooperation in the mycorrhizal symbiosis. Science 333 (6044): 880-882. DOI: 10.1126/science.1208473.

Lee PJ, Koske RE. 1994. Gigaspora gigantea seasonal abundance an aging of spores in a sand dune. Mycol Res 98: 453-457. DOI: 10.1016/S0953-7562(09)81203-3

Lehmann A, Rillig MC. 2015. Arbuscular mycorrhizal contribution to copper, manganese, and iron nutrient concentrations in crops - a metaanalysis. Soil Biol Biochem 81: 147-158. DOI: 10.1016/j.soilbio.2014.11.013.

Lennon JT, Jones SE. 2011. Microbial seed banks: the ecological and evolutionary implications of dormancy. Nat Rev Microbiol 9: 119130. DOI: $10.1038 /$ nrmicro2504.

Liu Y, Johnson NC, Mao L, Shi G, Jiang S, Ma X, Du G, An L, Feng H. 2015. Phylogenetic structure of arbuscular mycorrhizal community shifts in response to increasing soil fertility. Soil Biol Biochem 89: 196-205. DOI: 10.1016/j.soilbio.2015.07.007.

Lötter MC, Mucina L, Witkowski ETF. 2014. Classification of the indigenous forests of Mpumalanga Province, South Africa. S Afr J Bot 90: 37-51. DOI: 10.1016/j.sajb.2013.09.010.

Maltz MR, Treseder KK. 2015. Sources of inocula influence mycorrhizal colonization of plants in restoration projects: a meta-analysis. Restor Ecol 23: 625-634. DOI: 10.1111/rec.12231.

Martínez-García LB, Armas C, de Dios Miranda J, Padilla FM, Pugnaire FI. 2011. Shrubs influence arbuscular mycorrhizal fungi communitie in a semi-arid environment. Soil Biol Biochem 43 (3): 682-689. DOI: 10.1016/j.soilbio.2010.12.006
McKenzie NJ, Isbell RF, Jacquier DW, Brown KL. 2004. Australian soils and landscapes: an illustrated compendium. CSIRO Publishing, Canberra.

Morton JB. 1986. Three new species of Acaulospora (Endogonaceae) from high-aluminium, low pH soils in West Virginia. Mycologia 78: 641-648. DOI: 10.1080/00275514.1986.12025300.

Mucina L, Hoare DB, Lötter MC, du Preez PJ, Rutherford MC, ScottShaw CR, Bredenkamp GJ, Powrie LW, Scott L, Camp KGT, Cilliers SS, Bezuidenhout H, Mostert TH, Siebert SJ, Winter PJD, Burrows JE, Dobson L, Ward RA, Stalmans M, Oliver EGH, Siebert F, Schmidt E, Kobisi K, Kose L. 2006. Grassland Biome. In: Mucina L, Rutherford MC (eds). The vegetation of South Africa, Lesotho, and Swaziland. SANBI, Pretoria.

Nadian H. Smith SE, Alston AM, Murray RS, Siebert BD. 1998. Effects of soil compaction on phosphorus uptake and growth of Trifolium subterraneum colonized by four species of vesicular-arbuscular mycorrhizal fungi. New Phytol 140: 55-165. DOI: 10.1046/j.14698137.1998.00219.x

Olubode A, Babalola O, Dare M, Adeyemi NO, Aderibigbe S, Okonji C, Sakariyawo O. 2020. Diversity of indigenous arbuscular mycorrhizal fungi in rhizosphere of upland rice (Oryza sativa L.) varieties in Southwest Nigeria. Acta Fytotechn Zootechn 23 (2): 42-48. DOI: 10.15414/afz.2020.23.02.42-48

Peoples MB, Craswell ET. 1992. Biological nitrogen fixation: investments, expectations, and actual contributions to agriculture. Plant Soil 141 (1-2): 13-39. DOI: 10.1007/BF00011308.

Pielou EC. 1966. The measurement of diversity in different types of biological collections. J Theor Biol 13: 131-144. DOI: 10.1016/00225193(66)90013-0.

Read DJ. 1991. Mycorrhizas in ecosystem. Experientia 47 (4): 376-391. DOI: $10.1007 / \mathrm{BF} 01972080$

Rutherford MC, Westfall RH. 1986. The biomes of Southern Africa - an objective categorization. Mem Bot Survey South Africa 54: 1-98.

Santos-González JC, Nallanchakravarthula S, Alström S, Finlay RD. 2011. Soil, but not cultivar, shapes the structure of arbuscular mycorrhizal fungal assemblages associated with strawberry. Microb Ecol 62: 25-35. DOI: 10.1007/s00248-011-9834-7.

Schenck NC, Perez Y. 1990. Manual for the Identification of VA Mycorrhizal Fungi. Synergistic Publications. Gainesville, FL.

Schollenberger CJ, Simon RH. 1945. Determination of exchange capacity and exchangeable bases in soil-ammonium acetate method. J Soil Sc. Plant Nutr 59: 13-24. DOI: 10.1097/00010694-194501000-00004.

Shannon CE. 1948. The mathematical theory of communication. Bell Syst Tech J 27 (3): 379-423. DOI: 10.1002/j.1538-7305.1948.tb01338.x.

Singh S, Pandey A, Chaurasia B, Palni LMS. 2008. Diversity of arbuscular mycorrhizal fungi associated with the rhizosphere of tea growing in "natural" and "cultivated" ecosites. Biol Fertil Soils 44: 491-500. DOI: 10.1007/s00374-007-0231-9.

Smith SE, Facelli E, Pope S, Smith FA. 2010. Plant performance in stressful environments: interpreting new and established knowledge of the roles of arbuscular mycorrhizas. Plant Soil 326 (1-2): 3-20. DOI: 10.1007/s11104-009-9981-5.

Songachan LS, Kayang H. 2013. Diversity of arbuscular mycorrhizal fungi associated with Flemingia vestita Benth. ex Baker. Mycology 4 (2): 85-95. DOI: $10.1080 / 21501203.2013 .809026$.

Sonneveld C, Van den Ende J. 1971. Soil analysis by means of a 1:2 volume extract. Plant and Soil 35: 505-516. DOI: 10.1007/BF01372683.

Steinkellner S, Lendzemo V, Langer I, Schweiger P, Khaosaad T, Toussaint JP, Vierheilig H. 2007. Flavonoids and strigolactones in root exudates as signals in symbiotic and pathogenic plant-fungus interactions. Molecules 12 (7): 1290-1306. DOI: 10.3390/12071290.

Straker CJ, Hilditch AJ, Rey MEC. 2010. Arbuscular mycorrhizal fungi associated with cassava (Manihot esculenta Crantz) in South Africa. S Afr J Bot 76: 102-111. DOI: 10.1016/j.sajb.2009.09.005

Sweeney CJ, de Vries FT, van Dongen BE, Bardgett RD. 2021. Root traits explain rhizosphere fungal community composition among temperate grassland plant species. New Phytol 229: 1492-1507. DOI: 10.1111/nph.16976.

Toh SC, Lihan S, Yong BCW, Tiang BR, Abdullahi R, Edward R. 2018. Isolation and characterisation of arbuscular mycorrhizal (AM) fungi spores from selected plant roots and their rhizosphere soil environment. Malaysian J Microbiol 14 (4): 335-343. http://dx.doi.org/10.21161/mjm.144187.

Trappe JM. 1987. Phylogenetic and ecologic aspects of mycotrophy in the angiosperms from an evolutionary standpoint. In: Safir GR (ed). 
Ecophysiology of VA Mycorrhizal Plants, CRC Press, Boca Raton, FL.

Trytsman M, van Wyk AE, Masemola EL 2011. Systematics, diversity, and forage value of indigenous legumes of South Africa, Lesotho, and Swaziland. Afr J Biotechnol 10 (63): 13773-13779. DOI: 10.5897/AJB10.2224.

Walkley A, Black IA. 1934. An examination of Degtjareff method for determining soil organic matter and a proposed modification of the chromic acid titration method. Soil Sci 37: 29-37. DOI 10.1097/00010694-193401000-00003.

Wang FY, Liu RJ, Lin XG, Zhou JM. 2004. Arbuscular mycorrhizal status of wild plants in saline-alkaline soils of the Yellow River Delta. Mycorrhiza 14: 133-137. DOI: 10.1007/s00572-003-0248-3.

Wang J, Wang GG, Zhang B, Yuan Z, Fu Z, Yuan Y, Zhu L, Ma S, Zhang J. 2019. Arbuscular mycorrhizal fungi associated with tree species in a planted forest of Eastern China. Forests 10 (424): 1-14. DOI: 10.3390/f10050424.

Wang M, Jiang P. 2015. Colonization and diversity of AM fungi by morphological analysis on medicinal plants in southeast China. Sci World J 2015: 753842. DOI: 10.1155/2015/753842.
Weisany W, Raei Y, Allahverdipoor KH. 2013. Role of some of mineral nutrients in biological nitrogen fixation. Bull Environ Pharmacol Life Sci 2 (4): 77-84

Yahara T, Javadi F, Onoda Y, de Queiroz LP, Faith PD, Prado DE, Akasaka M, Kadoya T, Ishihama F, Davies S, Slik JWF, Yi T, Ma K, Bin C, Darnaedi D, Pennington RT, Tuda M, Shimada M, Ito M, Egan AN, Buerki S, Raes N, Kajita T, Vatanparast M, Mimura M, Tachida H, Iwasa Y, Smith GF, Victor JE, Nkonki T. 2013. Global legume diversity assessment: Concepts, key indicators, and strategies. Taxon 62 (2): 249-266. DOI: 10.12705/622.12.

Yano K, Yamauchi A, Iijima M, Kono Y. (1998). Arbuscular mycorrhizal formation in undisturbed soil counteracts compacted soil stress for pigeon pea. Appl Soil Ecol 10: 95-102. DOI: 10.1016/S09291393(98)00034-1.

Zhang Y, Guo LD, Liu RJ. 2004. Survey of arbuscular mycorrhizal fungi in deforested and natural forest land in the subtropical region of Dujiangyan, southwest China. Plant Soil 261: 257-263. DOI: 10.1023/B:PLSO.0000035572.15098.f6. 